STRUCTURAL BIOLOGY

ISSN 2059-7983

Received 23 March 2020

Accepted 11 January 2021

Edited by J. R. Helliwell, University of Manchester, United Kingdom

Keywords: lysozyme hydrolysis; hydrogen-bond network; high-resolution X-ray diffraction; neutron diffraction.

PDB references: lysozyme, complex with (GlcNAc) $)_{4}$, obtained in $\mathrm{H}_{2} \mathrm{O}, 7 \mathrm{br} 5$; obtained in $\mathrm{D}_{2} \mathrm{O}, 7$ deq; without ligand, 7der

Supporting information: this article has supporting information at journals.iucr.org/d

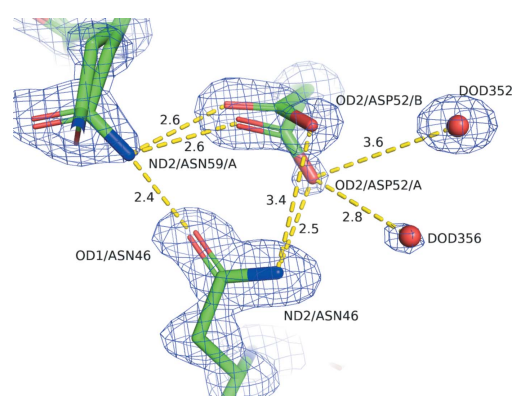
OPEN $\odot$ ACCESS

\section{Recent structural insights into the mechanism of lysozyme hydrolysis}

\author{
Ichiro Tanaka, ${ }^{\mathrm{a}, \mathrm{b} *}$ Ryota Nishinomiya, ${ }^{\mathrm{a}}$ Ryosuke Goto, ${ }^{\mathrm{a}}$ Shun Shimazaki ${ }^{\mathrm{c}}$ and \\ Toshiyuki Chatake $^{\text {d }}$
}

\begin{abstract}
${ }^{\mathbf{a}}$ Graduate School of Science and Engineering, Ibaraki University, Hitachi, Ibaraki 316-8511, Japan, ${ }^{\mathbf{b}}$ Frontier Research Center for Applied Atomic Sciences, Ibaraki University, Tokai, Ibaraki 319-1106, Japan, 'College of Engineering, Ibaraki University, Hitachi, Ibaraki 316-8511, Japan, and Institute for Integrated Radiation and Nuclear Science, Kyoto University, Kumatori, Osaka 590-0494, Japan. *Correspondence e-mail: ichiro.tanaka.h27@vc.ibaraki.ac.jp
\end{abstract}

Lysozyme hydrolyzes the glycosidic bonds between $N$-acetylmuramic acid and $N$-acetylglucosamine in peptidoglycans located in the bacterial cell wall. The mechanism of the hydrolysis reaction of lysozyme was first studied more than 50 years ago; however, it has not yet been fully elucidated and various mechanisms are still being investigated. One reaction system that has commonly been proposed is that the lysozyme intermediate undergoes covalent ligand binding during hydrolysis. However, these findings resulted from experiments performed under laboratory conditions using fluorine-based ligands, which facilitate the formation of covalent bonds between the ligands and the catalytic side chain of lysozyme. More recently, high-resolution X-ray structural analysis was used to study the complex of lysozyme with an $N$-acetylglucosamine tetramer. As a result, the carboxyl group of Asp52 was found to form a relatively strong hydrogen-bond network and had difficulty binding covalently to $\mathrm{C} 1$ of the carbohydrate ring. To confirm this hydrogen-bond network, neutron test measurements were successfully performed to a resolution of better than $1.9 \AA$.

\section{Introduction}

Lysozyme is a well known enzyme that hydrolyzes the glycosidic bonds between $N$-acetylmuramic acid and $N$-acetylglucosamine in peptidoglycans located in the bacterial cell wall. After the binding of the carbohydrate ring hexamer to the catalytic site of lysozyme, hydrolysis occurs between the fourth and fifth carbohydrate rings via two catalytic amino acids, such as Glu35 and Asp52 in the case of hen egg-white (HEW) lysozyme. Although the mechanism of the hydrolysis reaction of lysozyme was first investigated over 50 years ago (Phillips, 1967; Koshland, 1953; Imoto et al., 1972), a number of potential mechanisms are actively being studied. The reaction system that has most commonly been proposed is that the intermediate of lysozyme forms a covalent bond between the fourth carbohydrate ring and the side chain of Asp52 during hydrolysis (Vocadlo et al., 2001). However, these findings were obtained under laboratory conditions using fluorine-based ligands, which facilitate the formation of covalent bonds to the catalytic side chain of lysozyme. Furthermore, a shift of the carbohydrate chain to Asp52 was expected owing to distortion of the carbohydrate ring, forming a covalent bond through rotation of the side chain of Asp52. Many other structural analyses of lysozyme-carbohydrate complexes have been performed, such as complexes with tetra- $N$-acetylchitotetraose or a tetramer of $N$-acetyl-D-glucosamine [(GlcNAc $\left.)_{4}\right]$ (Maenaka et al., 1995; Yamada et al., 2015), as well as a complex with tri- $N$-acetylchitotetraose (Cheetham et al., 1992) 
and with a trimer of $\mathrm{N}$-acetyl-D-glucosamine $(\mathrm{GlcNAc})_{3}$ with moranoline at the end (Ogata et al., 2013). However, there is currently no clear evidence of covalent bonding between the fourth carbohydrate ring of (GlcNAc) 4 (NAG4) and the side chain of Asp52 during hydrolysis under natural conditions, whereas particular substrate analogs and Asp52 may easily form covalent bonds in an artificial manner. In addition, research has yet to consider the formation of a hydrogen-bond network around the catalytic amino acids using high-resolution data. Furthermore, turnover-rate experiments on mutated lysozyme indicated that most of the hydrolysis reaction did not take place via glycosyl formation (Abe et al., 2016).

The hydrogen-bond network needs to be characterized to determine whether or not rotation occurs. To this end, highresolution X-ray and neutron diffraction are indispensable tools, the latter of which is one of the best and most powerful methods for determining protein structures, including $\mathrm{H}$ atoms, unambiguously (Niimura \& Podjarny, 2011). Two neutron studies have investigated HEW lysozyme (Niimura et al., 1997; Bon et al., 1999); however, these did not contain ligands. Diffraction experiments using new-generation neutron sources can potentially characterize these structures (Tanaka et al., 2020).

In this study, the hydrogen-bond networks of a lysozymecarbohydrate complex were analyzed using high-resolution $\mathrm{X}$-rays to investigate and confirm the hydrolysis mechanism of this complex. Neutron test experiments were also performed to confirm the resolution of data from large crystals grown in $\mathrm{D}_{2} \mathrm{O}$ buffer. Experiments were conducted at the optimal $\mathrm{pH}$ $(\sim 5)$ of the enzyme using natural enzymatic products $\left[(\mathrm{GlcNAc})_{4}\right.$ as a product from $\left.(\mathrm{GlcNAc})_{6}\right]$ to provide a snapshot of the catalytic reaction intermediates.

\section{Materials and methods}

\subsection{Sample crystallization for X-ray and neutron diffraction}

HEW lysozyme was purchased from Sigma-Aldrich, St Louis, Missouri, USA (catalog No. L6876). (GlcNAc) 3 and $(\mathrm{GlcNAc})_{4}$ were purchased from Dextra Laboratories, Reading, England (catalog Nos. C8003 and C8004, respectively). (GlcNAc) 4 was also purchased from Megazyme, Bray, Ireland (catalog No. O-CHI4). The proteins and carbohydrates were used without further purification for X-ray structural analysis. Protein solutions were filtered using a $0.22 \mu \mathrm{m}$ filter to remove any impurities.

For X-ray diffraction, three types of crystals were used. A lysozyme-carbohydrate complex crystal was obtained in $\mathrm{H}_{2} \mathrm{O}$ from $6 \mu \mathrm{l}$ of a solution consisting of $40 \mathrm{mg} \mathrm{m}^{-1}$ lysozyme, $2.1 \mathrm{mg} \mathrm{ml}^{-1}$ (GlcNAc) $)_{4}$ and $0.7 \mathrm{M} \mathrm{NaCl}$ in $50 \mathrm{mM}$ sodium acetate $\mathrm{pH} 4.5$ buffer using the micro-batch method at $293 \mathrm{~K}$. A lysozyme-carbohydrate complex crystal was obtained in $\mathrm{D}_{2} \mathrm{O}$ from a mixture of $5 \mu \mathrm{l} 40 \mathrm{mg} \mathrm{ml}^{-1}$ lysozyme containing $2.1 \mathrm{mg} \mathrm{ml}^{-1}$ (GlcNAc) ${ }_{4}$ and $5 \mu \mathrm{l} 0.7 \mathrm{M} \mathrm{NaCl}$ in a $\mathrm{D}_{2} \mathrm{O}$ buffer of $50 \mathrm{~m} M$ sodium acetate $\mathrm{pD} 4.5$ by the hanging-drop method at $293 \mathrm{~K}$. The reservoir used was $0.7 \mathrm{M} \mathrm{NaCl}$ in a $\mathrm{D}_{2} \mathrm{O}$ buffer of $50 \mathrm{~m} M$ sodium acetate $\mathrm{pD}$ 4.5. A crystal of lysozyme without carbohydrate was obtained in $\mathrm{H}_{2} \mathrm{O}$ from $6 \mu \mathrm{l}$ of a solution consisting of $15 \mathrm{mg} \mathrm{ml}^{-1}$ lysozyme and $0.7 \mathrm{M} \mathrm{NaCl}$ in $50 \mathrm{~m} M$ sodium acetate $\mathrm{pH} 4.5$ using the micro-batch method at $293 \mathrm{~K}$.

For neutron diffraction, two complexes were prepared. A lysozyme-(GlcNAc) $)_{4}$ complex crystal was obtained from $20 \mu \mathrm{l}$ of a solution consisting of $15 \mathrm{mg} \mathrm{ml}^{-1}$ lysozyme, $1.0 \mathrm{mg} \mathrm{ml}^{-1}$ $(\mathrm{GlcNAc})_{4}, 0.25 \mathrm{M} \mathrm{NaCl}$ in a $\mathrm{D}_{2} \mathrm{O}$ buffer of $50 \mathrm{~m} M$ sodium acetate $\mathrm{pD} 4.5$ by the sitting-drop method using $1 \mathrm{ml}$ reservoir solution consisting of $0.5 \mathrm{M} \mathrm{NaCl}$ in the same buffer at $293 \mathrm{~K}$. A lysozyme-(GlcNAc) $)_{3}$ complex crystal was obtained from $10 \mu \mathrm{l}$ of a solution consisting of $17.5 \mathrm{mg} \mathrm{m}^{-1}$ lysozyme, $0.77 \mathrm{mg} \mathrm{ml}^{-1}$ (GlcNAc) $)_{3}, 0.6 \mathrm{M} \mathrm{NaCl}$ in a $\mathrm{D}_{2} \mathrm{O}$ buffer of $50 \mathrm{~m} M$ sodium acetate $\mathrm{pD} 4.5$ by the sitting-drop method with $0.5 \mathrm{ml}$ reservoir solution consisting of $1.2 \mathrm{M} \mathrm{NaCl}$ in the same buffer at $293 \mathrm{~K}$.

\subsection{X-ray diffraction experiments and analysis}

Prior to conducting the X-ray diffraction experiments, the crystals were flash-cooled in liquid nitrogen after soaking in $30 \%(v / v)$ glycerol buffer. The crystals were irradiated with $\mathrm{X}$-rays at a synchrotron facility (BL-5A at Photon Factory, KEK, Japan) under nitrogen gas at $100 \mathrm{~K}$ and a full data set was collected. HKL-2000 (Otwinowski \& Minor, 1997) or $X D S$ (Kabsch, 2010) were used to reduce the raw data, and Phenix (Liebschner et al., 2019) and Coot (Emsley et al., 2010) were used to analyze the structures. PDB entry 4wld (Yamada et al., 2015) was used as the initial model. The atoms were refined using the weight-optimization function included in phenix.refine, while anisotropic temperature factors were calculated. Figures were created using PyMOL (PyMOL Molecular Graphics System version 2.4; Schrödinger). The experimental conditions, data-reduction statistics and analyses are given in Table 1. The atomic coordinates and structural factors of the lysozyme complex in $\mathrm{H}_{2} \mathrm{O}$, the lysozyme complex in $\mathrm{D}_{2} \mathrm{O}$ and the lysozyme-only sample have been deposited in the Protein Data Bank as entries 7br5, 7deq and 7der, respectively.

With regard to the top difference-map peaks in the three models, we note the following: for the complex in $\mathrm{H}_{2} \mathrm{O}$ there is evidence of X-ray radiation damage to the Cys6-Cys127 disulfide, i.e. some movement of the $\mathrm{S}$ atoms in the disulfide, for the complex in $\mathrm{D}_{2} \mathrm{O}$ there is disorder at $\mathrm{Arg} 128$ that is not easy to model and is not pertinent to the subject of the study, and for lysozyme alone there is disorder in the sodium site environment.

\subsection{Neutron diffraction experiments}

Prior to conducting the neutron diffraction experiments, each crystal was placed in a quartz capillary after removing the buffer liquid from the crystal surface. The capillaries were sealed with a small amount of mother liquor to prevent the crystal from drying. The single crystals of the lysozyme$(\mathrm{GlcNAc})_{3}$ complex and the lysozyme-(GlcNAc) ${ }_{4}$ complex are shown in Fig. 1. The neutron experiments were conducted on BL03 (iBIX; Tanaka et al., 2010) of the Material and Life 
Science Facility (MLF) of J-PARC (Japan Proton Accelerator Research Complex) at $298 \mathrm{~K}$ with $400 \mathrm{~kW}$ proton power for $14 \mathrm{~h}$ in the case of the $(\mathrm{GlcNAc})_{3}$ complex and $500 \mathrm{~kW}$ proton power for $8 \mathrm{~h}$ in the case of the $($ GlcNAc) 4 complex. The beam cross section was $3 \mathrm{~mm}$ in diameter with a wavelength range of 2.18-6.18 .

\section{Results and discussion}

\subsection{X-ray structural analysis}

The crystal structures of two complexes in $\mathrm{D}_{2} \mathrm{O}$ and $\mathrm{H}_{2} \mathrm{O}$ and of lysozyme alone in $\mathrm{H}_{2} \mathrm{O}$ were determined using high-resolution data at 1.0 to $1.03 \AA$. Since there were no significant differences between the (GlcNAc) complex in $\mathrm{D}_{2} \mathrm{O}$ and in $\mathrm{H}_{2} \mathrm{O}$, the $\mathrm{D}_{2} \mathrm{O}$ complex structure will be referred to as the complex structure from this point in the discussion. At the ligand-binding site, (GlcNAc) ${ }_{4}$ was confirmed as a tetramer of GlcNAc (Fig. 2). Although the occupancy of the fourth residue of $($ GlcNAc) 4 (NAG4) was found to be relatively low $(0.36$ and 0.21 for the complexes in $\mathrm{D}_{2} \mathrm{O}$ and $\mathrm{H}_{2} \mathrm{O}$, respectively) compared with the other three NAGs, NAG4 can be readily observed in the $3 \sigma$ difference Fourier map in Fig. 2. In addition, NAG4 and four water molecules share the same site, with double conformations $\mathrm{A}$ and $\mathrm{B}$ with occupancies of 0.36 and 0.64 , respectively (Fig. 3). The four water molecules are well conserved among the structures of the complexes and of lysozyme alone.

When comparing the structure around the D-site carbohydrate (NAG4), four water molecules in the complex overlap with those in the structure without ligand, and these water
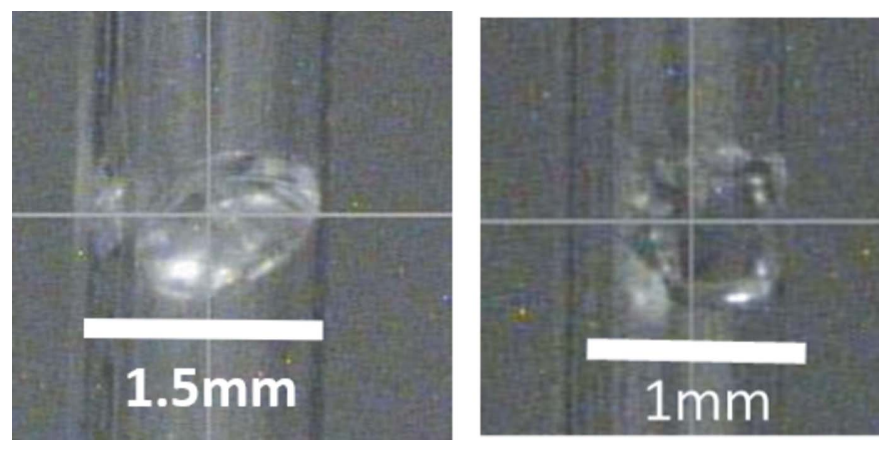

Figure 1

Single crystals of the lysozyme-(GlcNAc) $)_{3}$ complex (left; $1.1 \times 0.65 \times$ $0.4 \mathrm{~mm}, 0.29 \mathrm{~mm}^{3}$ volume) and the lysozyme-(GlcNAc) $)_{4}$ complex (right; $0.7 \times 0.7 \times 0.25 \mathrm{~mm}, 0.12 \mathrm{~mm}^{3}$ volume $)$ used in neutron diffraction experiments.

Table 1
Values in parentheses are for the outermost shell.

\begin{tabular}{|c|c|c|c|}
\hline PDB code & $7 \mathrm{br} 5$ & $7 \mathrm{deq}$ & $7 \mathrm{der}$ \\
\hline Notes & Complex in $\mathrm{H}_{2} \mathrm{O}$ & Complex in $\mathrm{D}_{2} \mathrm{O}$ & No ligand in $\mathrm{H}_{2} \mathrm{O}$ \\
\hline Diffraction source & BL-5A, Photon Factory & BL-5A, Photon Factory & BL-5A, Photon Factory \\
\hline Wavelength $(\AA)$ & 1.00 & 1.00 & 1.00 \\
\hline Temperature (K) & 100 & 100 & 100 \\
\hline Space group & $P 4_{3} 2_{1} 2$ & $P 4_{3} 2_{1} 2$ & $P 4_{3} 2_{1} 2$ \\
\hline$a, c(\AA)$ & $77.309,38.214$ & $77.029,38.285$ & $78.777,37.093$ \\
\hline Resolution range $(\AA)$ & $16.83-1.00(1.036-1.000)$ & $34.45-1.03(1.067-1.030)$ & $33.56-1.03(1.067-1.030)$ \\
\hline Total reflections & 906663 (87281) & 706539 (62633) & 713001 (63054) \\
\hline Unique reflections & 61939 (6187) & $57379(5652)$ & $58114(5713)$ \\
\hline Multiplicity & $14.6(14.1)$ & $12.3(11.1)$ & $12.3(11.0)$ \\
\hline Completeness (\%) & $98.53(99.95)$ & $100.00(100.00)$ & $99.99(100.00)$ \\
\hline$R_{\text {merge }}$ & $0.07595(0.747)$ & $0.06526(1.274)$ & $0.07402(0.6635)$ \\
\hline$\langle I / \sigma(I)\rangle$ & $40.20(4.38)$ & $22.17(2.21)$ & $19.51(2.25)$ \\
\hline $\mathrm{CC}_{1 / 2}$ & $0.997(0.893)$ & $0.999(0.837)$ & $0.997(0.929)$ \\
\hline$R_{\text {work }} / R_{\text {free }}$ & $0.1392 / 0.1669$ & $0.1325 / 0.1485$ & $0.1487 / 0.1733$ \\
\hline \multicolumn{4}{|l|}{ No. of non-H atoms } \\
\hline Total & 1343 & 1362 & 1285 \\
\hline Protein & 1081 & 1078 & 1036 \\
\hline Ligand/ion & 62 & 62 & 5 \\
\hline Water & 193 & 210 & 233 \\
\hline \multicolumn{4}{|l|}{$B$ factor $\left(\AA^{2}\right)$} \\
\hline Overall & 18.00 & 16.90 & 17.70 \\
\hline Protein & 15.10 & 13.80 & 14.70 \\
\hline Ligand/ion & 20.70 & 18.00 & 17.10 \\
\hline Water & 32.90 & 31.70 & 30.60 \\
\hline \multicolumn{4}{|l|}{ R.m.s.d. from ideality } \\
\hline Bond lengths $(\AA)$ & 0.006 & 0.006 & 0.006 \\
\hline Bond angles $\left({ }^{\circ}\right)$ & 1.10 & 1.08 & 1.12 \\
\hline \multicolumn{4}{|l|}{ Ramachandran plot } \\
\hline Most favored (\%) & 99 & 99 & 99 \\
\hline Allowed (\%) & 1 & 1 & 1 \\
\hline
\end{tabular}

molecules and NAG4 share a similar site with double conformations as described previously. The catalytic residue Asp52 in the complex structure has also double conformations $\mathrm{A}$ and $\mathrm{B}$, with occupancies of 0.44 and 0.56 , respectively. The corresponding residue of lysozyme alone overlaps with the $\mathrm{B}$ conformation of the complex, which is closer to the NAG4 site (Fig. 3). Based on these findings, approximately $40 \%$ of the crystal molecules have NAG4 and Asp52 orientations that are distant from the NAG4 site (A conformation) due to ligand

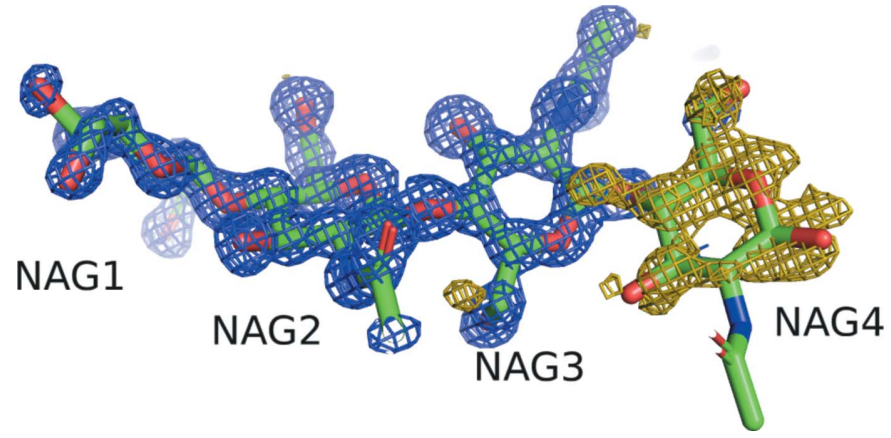

Figure 2

Stick structure of (GlcNAc) $)_{4}$ bound to lysozyme in $\mathrm{D}_{2} \mathrm{O}$ with electrondensity maps (green, carbon; red, oxygen; blue, nitrogen). NAG1, NAG2, NAG3 and NAG4 correspond to the first, second, third and fourth residue of (GlcNAc $)_{4}$, respectively. The blue and olive electron-density maps are $2 F_{\mathrm{o}}-F_{\mathrm{c}}$ at $1.5 \sigma$ and $F_{\mathrm{o}}-F_{\mathrm{c}}$ at $3.0 \sigma$, respectively, which were drawn by omitting NAG4. 
binding. NAG4 may be partly hydrolyzed during the crystallization process.

The distance between $\mathrm{C} 1$ of NAG4 and OD2 of Asp52 was examined. The distances are 4.0 and $3.3 \AA$ for the A conformation, where the carbohydrate was bound to the $\mathrm{D}$ site, and for the B conformation, where no ligand was bound, respectively (Fig. 4). The distance appears to be too great for Asp52 to bind to $\mathrm{C} 1$ of NAG4; it can be thought that the A conformation is close to the hydrolysis intermediate.

The hydrogen-bond network around Asp52 was also examined (Fig. 5). A relatively solid hydrogen-bonding polygon is formed by Asp52 and Asn59 in the A conformation and Asn46. The hydrogen bonds, with distances of 2.2-2.6 can be categorized as moderately strong (Jeffery, 1997). Additionally, OD2 of Asp52 makes bifurcated hydrogen bonds to DOD356 and DOD352 with distances of 2.8 and 3.6 A, respectively. Based on this, OD2 of Asp52 in the A conformation appears to have difficulty in forming a covalent bond to $\mathrm{C} 1$ of NAG4.

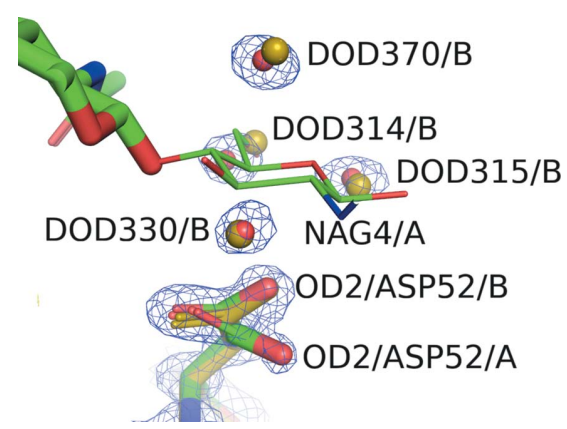

Figure 3

Superimposed stick structures of the lysozyme-(GlcNAc) $)_{4}$ complex (CPK colors with green for carbon; complex in $\mathrm{D}_{2} \mathrm{O}$ ) and of lysozyme without ligands (olive; lysozyme alone) where only NAG4 is drawn as a wire model for clarity. $\mathrm{O}$ atoms of water molecules both in lysozyme alone and in the complex in $\mathrm{D}_{2} \mathrm{O}$ are illustrated as olive and red balls, respectively. NAG4/A and OD2/ASP52/A are in the A conformation. In contrast, four water molecules and OD2/ASP52/B are located in the B conformation. OD2 of Asp52 in lysozyme alone overlaps with OD2/ASP52/B. The blue contour lines correspond to $2 F_{\mathrm{o}}-F_{\mathrm{c}}$ maps at $1.5 \sigma$ for the complex in $\mathrm{D}_{2} \mathrm{O}$, except for $(\mathrm{GlcNAc})_{4}$.

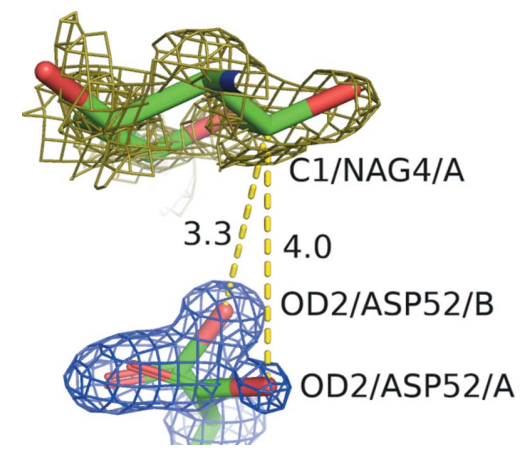

Figure 4

The distances (labeled in $\AA$ ) between C1/NAG4/A and OD2/ASP52 in the $\mathrm{A}$ and $\mathrm{B}$ conformations are shown as yellow dashed lines. The blue and olive contour lines represent $2 F_{\mathrm{o}}-F_{\mathrm{c}}$ maps at $1.5 \sigma$ and $0.6 \sigma$ for the complex in $\mathrm{D}_{2} \mathrm{O}$, respectively. Atoms are shown as sticks (green, carbon; red, oxygen; blue, nitrogen).
In the present complexes, two conformations are observed in the active site of the lysozyme molecule. The two conformations suggest that the present crystal structure may be a mixture of the lysozyme-first product (or new substrate) complex (A conformation) and the lysozyme-second product complex after the chemical reaction (B conformation). This is the first time that such a structure with co-existing conformations has been observed. This provides evidence that $(\mathrm{GlcNAc})_{4}$ was hydrolyzed to $(\mathrm{GlcNAc})_{3}$ at some stage.

In the present analysis, the native substrate is used. Interestingly, a covalent bond between lysozyme and (GlcNAc) $)_{4}$ is not observed, unlike in previous artificial complexes (Vocadlo et al., 2001; Ogata et al., 2013), suggesting the possibility of another hydrolysis mechanism differing from that via a glycosyl intermediate.

Instead of a covalent bond, the hydrogen-bond network differs between lysozyme complexes with the ligand and lysozyme without substrate. In order to obtain further detailed information, a neutron diffraction experiment is now needed to determine the hydrogen positions of the lysozyme, substrate and water molecules.

\subsection{Neutron test experiment}

Prior to performing the neutron diffraction experiment, tests were conducted to determine whether the quality and volume of the crystals were sufficient for diffraction at a suitable resolution to observe the $\mathrm{H}$ atoms in the molecular structure. For both the lysozyme-(GlcNAc) $)_{4}$ and lysozyme$(\mathrm{GlcNAc})_{3}$ complexes, diffraction was successfully obtained to resolutions of 1.87 and $1.89 \AA$, respectively. At these resolutions, the orientations of the water molecules and the $\mathrm{H}$ atoms should be identifiable. For X-ray/neutron refinement, ambient temperature data need to be obtained using X-ray diffraction

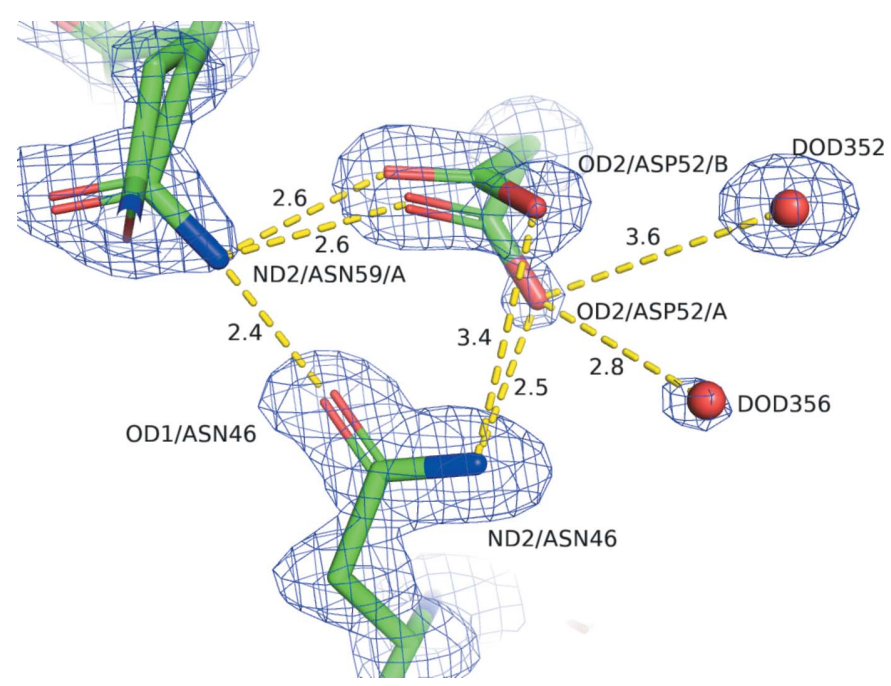

Figure 5

Hydrogen-bond network around Asp52. Yellow dashed lines represent hydrogen bonds or other interactions. The numbers denote the distances in $\AA$. Atoms are shown as sticks (green, carbon; red, oxygen; blue: nitrogen). The $\mathrm{O}$ atom of the water molecule is shown as a red sphere. The electron-density maps are shown as blue contour lines for the protein $\left(2 F_{\mathrm{o}}-F_{\mathrm{c}}\right.$ map at $\left.1.5 \sigma\right)$. 
at a comparable resolution using the same crystals as those used for neutron full data measurements.

\section{Conclusion}

A high-resolution structural analysis of lysozyme complexed with an $\mathrm{N}$-acetylglucosamine tetramer [(GlcNAc $\left.)_{4}\right]$ was successfully performed using X-ray diffraction. Compared with the structure of lysozyme alone, it was found that NAG4 shares its site with four water molecules that were well conserved in the structure of lysozyme alone. A relatively strong hydrogen-bond network was observed around the catalytic residues, specifically Asp52, and the distance between C1 of NAG4 and OD2 of Asp52 was observed to be $4.0 \AA$. These results indicate that it may be difficult for lysozyme to hydrolyze carbohydrate molecules via the glycosyl intermediate. To confirm the structure of this hydrogen-bond network, neutron diffraction test experiments were performed at resolutions that will permit us to observe the orientations of both water molecules and $\mathrm{H}$ atoms in a full neutron data collection.

\section{Acknowledgements}

The authors would like to thank Professor K. Igarashi (University of Tokyo) for fruitful discussions on hydrolysis. Synchrotron-radiation experiments were conducted on BL-5A of the Photon Factory, KEK, Ibaraki, Japan (proposal Nos. 2014G650, 2016G594, 2017G650, 2018G645 and 2019G657) and the neutron experiments were conducted on BL03 of MLF, J-PARC, Japan (proposal Nos. 2016PX0003, 2017PX0015 and 2019B0294).

\section{Funding information}

This study was partly supported by the Ibaraki Prefectural Government and JSPS KAKENHI Grant No. JP20H05436 (IT).

\section{References}

Abe, Y., Kubota, M., Takazaki, S., Ito, Y., Yamamoto, H., Kang, D., Ueda, T. \& Imoto, T. (2016). Protein Sci. 25, 1637-1647.

Bon, C., Lehmann, M. S. \& Wilkinson, C. (1999). Acta Cryst. D55, 978-987.

Cheetham, J. C., Artymiuk, P. J. \& Phillips, D. C. (1992). J. Mol. Biol. 224, 613-628.

Emsley, P., Lohkamp, B., Scott, W. G. \& Cowtan, K. (2010). Acta Cryst. D66, 486-501.

Imoto, T., Johnson, L. N., North, A. C. T., Phillips, D. C. \& Rupley, J. A. (1972). The Enzymes, 3rd ed., edited by P. D. Boyer, Vol. 7, pp. 665-868. New York: Academic Press.

Jeffery, G. A. (1997). An Introduction to Hydrogen Bonding. Oxford University Press.

Kabsch, W. (2010). Acta Cryst. D66, 125-132.

Koshland, D. E. (1953). Biol. Rev. 28, 416-436.

Liebschner, D., Afonine, P. V., Baker, M. L., Bunkóczi, G., Chen, V. B., Croll, T. I., Hintze, B., Hung, L.-W., Jain, S., McCoy, A. J., Moriarty, N. W., Oeffner, R. D., Poon, B. K., Prisant, M. G., Read, R. J., Richardson, J. S., Richardson, D. C., Sammito, M. D., Sobolev, O. V., Stockwell, D. H., Terwilliger, T. C., Urzhumtsev, A. G., Videau, L. L., Williams, C. J. \& Adams, P. D. (2019). Acta Cryst. D75, 861-877.

Maenaka, K., Matsushima, M., Song, H., Sunada, F., Watanabe, K. \& Kumagai, I. (1995). J. Mol. Biol. 247, 281-293.

Niimura, N., Minezaki, Y., Nonaka, T., Castagna, J.-C., Cipriani, F., Høghøj, P., Lehmann, M. S. \& Wilkinson, C. (1997). Nat. Struct. Biol. 4, 909-914.

Niimura, N. \& Podjarny, A. (2011). Neutron Protein Crystallography: Hydrogen, Proton, and Hydration in Bio-macromolecules. Oxford University Press.

Ogata, M., Umemoto, N., Ohnuma, T., Numata, T., Suzuki, A., Usui, T. \& Fukamizo, T. (2013). J. Biol. Chem. 288, 6072-6082.

Otwinowski, Z. \& Minor, W. (1997). Methods Enzymol. 276, 307-326.

Phillips, D. C. (1967). Proc. Natl Acad. Sci. USA, 57, 483-495.

Tanaka, I., Chatake, T., Fujiwara, S., Hosoya, T., Kusaka, K., Niimura, N., Yamada, T. \& Yano, N. (2020). Methods Enzymol. 634, 101-123. Tanaka, I., Kusaka, K., Hosoya, T., Niimura, N., Ohhara, T., Kurihara, K., Yamada, T., Ohnishi, Y., Tomoyori, K. \& Yokoyama, T. (2010). Acta Cryst. D66, 1194-1197.

Vocadlo, D. J., Davies, G. J., Laine, R. \& Withers, S. G. (2001). Nature, 412, 835-838.

Yamada, H., Nagae, T. \& Watanabe, N. (2015). Acta Cryst. D71, 742753. 